ist mit fast jedem Endgerät direkt nutzbar - auch mit Tablets oder Smartphones.

Kliniken, die bereits über eine GUSbox 19" als KV-SafeNet-Zugangsgerät verfügen, können den Netzkopplungsdienst einfach per Update nachrüsten.

Die Industrieausführung der GUSbox-Gerätefamilie ist mit ihrem 19-Zoll-Standardformat auf die IT-Anforderungen professioneller Rechenzentren zugeschnitten und zudem nutzbar mit jedem Krankenhausinformationssystem (KIS).

\section{DsiN-Cloud-Scout: Herstellerunabhängiger und produktneutraler Online-Check für Unternehmen}

Unter dem Dach von Deutschland sicher im Netz e.V. (DsiN) wurde ein DsiN-Cloud-Scout entwickelt, der kleinen und mittelständischen Unternehmen eine wichtige Orientierungshilfe bietet und dabei wirtschaftliche, technische und rechtliche Aspekte abdeckt. Die Nutzer erhalten auf www.DsiN-Cloud-Scout.de in 10 bis $15 \mathrm{Mi}-$ nuten eine individuelle Auswertung und Empfehlung zur Nutzung von Cloud Computing mit Fokus auf IT-Sicherheit und Datenschutz. Inhaltliche Schwerpunkte des neuen DsiN-Cloud-Scouts sind beispielsweise Berechtigungskonzepte, integrierte Sicherheitslösungen, Schutz der Zugriffsgeräte, Applikationssicherheit und Bedingungen an den Internetzugang - Themen mit denen sich Unternehmen beschäftigen sollten, bevor sie Cloud-Anwendungen in die bestehende Unternehmensinfrastruktur einbinden. Das Informationsangebot gibt Unternehmen einen ersten Überblick über die Anforderungen an Cloud Computing, ersetzt aber keine individuelle Sicherheitsanalyse. Der DsiN-Cloud-Scout wurde von DsiN gemeinsam mit seinen Mitgliedern Deutsche Telekom AG, Microsoft Deutschland GmbH und der SAP AG erstellt.

Oliver Bussmann, stellvertretender DsiN-Vorstandsvorsitzender und Chief Information Officer von der SAP AG, erklärte: „Cloud Computing bietet für viele Mittelständler eine Chance: Sie können zu günstigen Preisen moderne IT-Systeme nutzen, ohne selbst entsprechendes Fachpersonal beschäftigen zu müssen. Grundlage für eine fundierte Entscheidung pro oder contra Cloud Computing ist das Wissen um die Chancen und Risiken. Genau hier setzt der DsiNCloud-Scout an."

Eine Umfrage von DsiN unter 375 mittelständischen Unternehmen hat eine deutliche Unsicherheit im Hinblick auf die rechtsund datenschutzkonforme Nutzung von Cloud Computing ergeben: Von den 16\% der Unternehmen, die bereits mit der Cloud arbeiten, kennen 19\% die Sicherheitsanforderungen und rechtlichen Rahmenbedingungen überhaupt nicht, $42 \%$ kennen sie nur teilweise. Von den Unternehmen, die sich mit der Cloud-Nutzung beschäftigen (24\%), kennen nur 13\% die Sicherheitsanforderungen, $57 \%$ der Befragten sind sie teilweise bekannt und $30 \%$ wissen nichts darüber.

\section{De-Mail in der Bundesverwaltung: Empfehlungen des BfDI}

Der Bundesbeauftragte für den Datenschutz und die Informationsfreiheit Peter Schaar hat am 01.03.2013 Empfehlungen zur sicheren Nutzung von De-Mail veröffentlicht.

Peter Schaar: „De-Mail bietet - anders als die normale E-Mail die Chance, Informationen gesichert zu übertragen. Damit können die meisten Kommunikationsvorgänge zwischen Verwaltung und Bürgerinnen und Bürgern endlich angemessen geschützt werden. Allerdings sind Nutzer und Anbieter bislang noch verunsichert, inwieweit auch besonders sensible Inhalte mit De-Mail versandt werden können. Mit diesen Empfehlungen möchte ich über den sicheren Einsatz von De-Mail informieren."

Nur eine „Ende-zu-Ende-Verschlüsselung" schließe völlig aus, so Schaar, dass ein Dritter vom Inhalt der De-Mail Kenntnis erlangen kann. Eine entsprechende Forderung der Datenschutzbeauftragten des Bundes und der Länder wurde aber im Gesetzgebungsverfahren nicht aufgegriffen.

Mit seinen Empfehlungen informiert der Bundesdatenschutzbeauftragte Bürgerinnen und Bürger sowie Bundesbehörden, wie der Versand besonders schützenswerter Daten mittels De-Mail datenschutzgerecht erfolgen kann. Behörden und andere Institutionen, die untereinander personenbezogene Daten versenden, sollen DeMails stets Ende-zu-Ende verschlüsseln. In der Bürger-Kommunikation kann dagegen eine Risikoabschätzung Aufschluss darüber geben, ob De-Mail auch ohne diese zusätzliche Schutzmaßnahme verwendet werden kann. Außerdem soll dieser Kommunikationsweg nur dann genutzt werden dürfen, wenn Bürgerinnen und Bürger den Zugang auch eindeutig eröffnet haben. Die Handreichung ist auf der Internetseite des Bundesdatenschutzbeauftragten veröffentlicht: www.datenschutz.bund.de.

De-Mail soll eine einfache und sichere E-Mail-Kommunikation ermöglichen. Das zugrunde liegende Gesetz ist im Mai 2011 in Kraft getreten. Die ersten Anbieter gibt es seit knapp einem Jahr. DeMail-Anbieter müssen eine Akkreditierung beim Bundesamt für Sicherheit in der Informationstechnik und eine Datenschutzzertifizierung beim Bundesdatenschutzbeauftragten durchlaufen.

Hier finden Sie die Empfehlungen als Download: http://www. bfdi.bund.de/SharedDocs/Publikationen/Sachthemen/DEMail/DeMailHandreichung.pdf?_blob=publicationFile

\section{HmbBfDI: Bußgeld gegen Google festgesetzt}

Der Hamburgische Beauftragte für Datenschutz und Informationsfreiheit (HmbBfDI) hat am 22.04.2013 gegen Google Inc. wegen unzulässiger WLAN-Mitschnitte ein Bußgeld von 145.000 Euro verhängt.

Von 2008 bis 2010 fotografierte Google nicht nur Straßen und Häuser für den Dienst Google Street View, sondern erfasste zugleich auch WLAN in Reichweite der dabei verwendeten Fahrzeuge. Dabei wurden, wie Google auf Nachfrage des Datenschutzbeauftragten einräumte, auch Inhaltsdaten der erfassten unverschlüsselten WLAN-Anschlüsse aufgezeichnet.

Dies bestätigte auch die Auswertung der von Google in Kopie zur Untersuchung des Sachverhalts zur Verfügung gestellten Daten. Unter den im Vorbeifahren erfassten Informationen befanden sich auch erhebliche Mengen an personenbezogenen Daten unter- 